\title{
An Elementary Construction on Nonlinear Coherent States Associated to Generalized Bargmann Spaces
}

\author{
Abdelkader Intissar ${ }^{1,2}$ \\ ${ }^{1}$ Equipe d'Analyse spectrale, UMR-CNRS n : 6134, Université de Corse, \\ Quartier Grossetti, 20250 Corté, France \\ ${ }^{2}$ Le Prador, 129 rue du Commandant Rolland, 13008 Marseille, France \\ Correspondence should be addressed to Abdelkader Intissar, intissar@univ-corse.fr
}

Received 27 July 2009; Revised 28 December 2009; Accepted 11 February 2010

Academic Editor: Ricardo Estrada

Copyright (C 2010 Abdelkader Intissar. This is an open access article distributed under the Creative Commons Attribution License, which permits unrestricted use, distribution, and reproduction in any medium, provided the original work is properly cited.

Consider the space $L_{2}(\mathbb{C}, d \mu(z))$, where $d \mu(z)=e^{-|z|^{2}} d z \wedge d \bar{z}$ is the Gaussian measure, and its generalized Bargmann subspaces $E_{m}$ which are the null kernels of the operator $\Delta=-\partial^{2} / \partial z \partial \bar{z}+$ $\bar{z}(\partial / \partial \bar{z})-m I ; m=0,1, \ldots$ In this work, we present an other construction of $E_{m}$ following the Hermite functions which allows us to define a family of generalized Bargmann transform $B_{m}$ which maps isometrically $E_{m}$ into $L_{2}(\mathbb{R})$. The generalized coherent states $|z\rangle_{m}$ associated to $E_{m}$ are constructed and some properties of them are given.

\section{Introduction}

The annihilation operator $a$ and the creator operator $a^{*}$ are well-known from the quantum theory of harmonic oscillator. They are defined by the commutation relation

$$
\left[a, a^{*}\right]=1
$$

Of special interest is a representation of these operators $a$ and $a^{*}$ as linear operators in a separable Hilbert $H$ spanned by the eigenvectors $|n\rangle ; n=0,1, \ldots$ of the positive semidefinite number operator $N=a^{*} a$.

One has the well-known relations

$$
a|n\rangle=\sqrt{n}|n-1\rangle, \quad a^{*}|n\rangle=\sqrt{n+1}|n+1\rangle .
$$


The coherent states analysis is a very well-known tool in physics, in particular in quantum optics and in quantum mechanics. The name "coherent states" was first used by Glauber, Nobel prize in physics (2005), for his works in quantum optics and electrodynamics. In the book of Klauder and Skagerstam [1], the reader can get an idea of the coherent states application fields in physics and mathematical-physics.

A general mathematical theory of coherent states is detailed in [2].

Let us begin by reviewing the most important properties of the space $\mathbf{I}_{0}$ of coherent states spanned by the set $\left\{|z\rangle_{0}\right\}$, where

$$
|z\rangle_{0}=e^{-1 / 2|z|^{2}} \sum_{n=0}^{\infty} \frac{z^{n}}{\sqrt{n !}}|n\rangle
$$

for each complex $z=x+i y$.

(i) The vectors $|z\rangle_{0}$ are the eigenvectors of the annihilation operator; one has $a|z\rangle_{0}=$ $z|z\rangle_{0}$.

(ii) The space $\mathbf{I}_{0}$ of coherent states is a separable Hilbert space which is isomorphic to the Hilbert space $H$ spanned by $\{|n\rangle\}$.

(iii) $|n\rangle=a^{*^{n}} / \sqrt{n !}|0\rangle$.

(iv) $|z\rangle_{0}=e^{-|z|^{2} / 2} e^{z a^{*}}|0\rangle$.

(v) $0\langle z|=\langle 0| e^{\bar{z} a} e^{-|z|^{2} / 2}$ is the adjoint vector of coherent state $|z\rangle_{0}$, where $\bar{z}=x-i y$.

From the above properties, we obtain

(vi) $a^{*}|z\rangle_{0}=(\bar{z} / 2+\partial / \partial z)|z\rangle_{0}$,

(vii) $a|z\rangle_{0}=(z / 2-\partial / \partial \bar{z})|z\rangle_{0}$,

where $\partial / \partial z$ and $\partial / \partial \bar{z}$ are the linear partial differential operators on $\mathbb{R}^{2}$ given by

$$
\begin{array}{ll}
\frac{\partial}{\partial z}=\frac{\partial}{\partial x}-i \frac{\partial}{\partial y}, & z=x+i y \\
\frac{\partial}{\partial \bar{z}}=\frac{\partial}{\partial x}+i \frac{\partial}{\partial y}, & \bar{z}=x-i y .
\end{array}
$$

Let $Z=\partial / \partial z+\bar{z} / 2$ and $\bar{Z}=\partial / \partial \bar{z}-z / 2$

Then we define the linear partial differential operator $L$ on $\mathbb{R}^{2}$ by

$$
L=-\frac{1}{2}(Z \bar{Z}+\bar{Z} Z)
$$

The vector fields $Z$ and $\bar{Z}$ and the identity operator form a basis for a Lie algebra in which the Lie bracket of two elements is their commutator. In fact, $-\bar{Z}$ is the formal adjoint of $\mathrm{Z}$ and $L$ is an elliptic partial differential operator on $\mathbb{R}^{2}$ given by

$$
L=-\left(\frac{\partial^{2}}{\partial x^{2}}+\frac{\partial^{2}}{\partial y^{2}}\right)+\frac{1}{4}\left(x^{2}+y^{2}\right)-i\left(x \frac{\partial}{\partial y}-y \frac{\partial}{\partial x}\right)
$$


Thus, $L$ is the usual Hermite operator

$$
-\left(\frac{\partial^{2}}{\partial x^{2}}+\frac{\partial^{2}}{\partial y^{2}}\right)+\frac{1}{4}\left(x^{2}+y^{2}\right)
$$

perturbed by the rotation operator

$$
-i\left(x \frac{\partial}{\partial y}-y \frac{\partial}{\partial x}\right)
$$

The strongly continuous one parameter semigroup $e^{-t L}, t>0$ is ultracontractive in the sense that for any $t>0, e^{-t L}$ is a bounded linear operator from $L_{2}\left(\mathbb{R}^{2}\right)$ to $L_{\infty}\left(\mathbb{R}^{2}\right)$, and it is hypercontractive in the sense that for all $t>0, e^{-t L}$ is a bounded linear operator from $L_{2}\left(\mathbb{R}^{2}\right)$ to $L_{4}\left(\mathbb{R}^{2}\right)$.

The theory of ultracontractive semigroups can be found in Davie's book [3] and the connections of hypercontractivity with constructive quantum field theory are attributed to Nelson [4] and are explained in the Simon's book [5].

Coming back to the space $\mathbf{I}_{0}$ of coherent states, it is closely related to Bargmann's space which was used in [6] for the canonical commutation rules as representation space of quantum mechanics. Since then, it had occurred in many different contents, that is, in representation theory of nilpotent Lie groups and in Reggeon field theory. This last theory is governed by a nonselfadjoint Gribov operator $[7,8]$ constructed as a polynomial in $a$ and $a^{*}$.

For any $\left|\phi_{0}\right\rangle \in \mathbf{I}_{0}$, we can define an entire analytic function by

$$
\phi_{0}(z)=e^{1 / 2|z|^{2}}\left\langle\phi_{0} \mid z\right\rangle_{0}=\sum_{n=0}^{\infty} \frac{z^{n}}{\sqrt{n !}}\left\langle\phi_{0} \mid n\right\rangle .
$$

As $\int_{\mathbb{C}}\left|\left\langle\phi_{0} \mid z\right\rangle_{0}\right|^{2} d x d y\langle\infty \cdot$ then

$$
\int_{\mathbb{C}}\left|\phi_{0}(z)\right|^{2} e^{-|z|^{2}} d x d y<\infty
$$

We denote the Bargmann space by:

$$
E_{0}=\left\{\phi_{0}: \mathbb{C} \longrightarrow \mathbb{C} \text { entire; } \int_{\mathbb{C}}\left|\phi_{0}(z)\right|^{2} e^{-|z|^{2}} d x d y<\infty\right\}
$$

$E_{0}$ is closed in $L_{2}(\mathbb{C}, d \mu(z))$, where the measure $d \mu(z)=e^{-|z|^{2}} d x d y$ and is closed related to $L_{2}(\mathbb{R})$ by an unitary transform of $L_{2}(\mathbb{R})$ onto $E_{0}$ given in [6] by the following integral transform (for some appropriate constant $c$ ):

$$
\phi_{0}(z)=\left[B_{0} f\right](z)=c \int_{\mathbb{R}} e^{-(1 / 2) z^{2}+\sqrt{2} u z-(1 / 2) u^{2}} f(u) d u
$$

If $f \in L_{2}(\mathbb{R})$, the integral converges absolutely. 
In $L_{2}(\mathbb{C}, d \mu(z))$, Askour et al. [9, 10] have introduced the so-called"generalized" Bargmann spaces $E_{m}, m=0,1, \ldots$, as null kernels of the operator

$$
\Delta=-\frac{\partial^{2}}{\partial z \partial \bar{z}}+\bar{z} \frac{\partial}{\partial \bar{z}}-m I, \quad m=0,1, \ldots
$$

and have proved that $L_{2}(\mathbb{C}, d \mu(z))$ is the direct sum of the Bargmann space $E_{0}$ and all "generalized" Bargmann spaces $E_{m}$.

Otherwise, Vasilevski in [11] concerning the structure of polyanalytic Fock spaces has introduced the so-called "true-poly-Fock" spaces and proved that $L_{2}(\mathbb{C}, d \mu(z))$ is the direct sum of the Bargmann space (Fock) and all "true-poly-Fock" spaces.

According to chapter 2 of Daubechies book [12], we give in this work a family of embedding operators $B_{m}$ from $L_{2}(\mathbb{R})$ into $L_{2}(\mathbb{C}, d \mu(z))$ such that the range $B_{0}\left(L_{2}(\mathbb{R})\right)$ is a Bargmann space and $B_{m}\left(L_{2}(\mathbb{R})\right)$ are reproducing subspaces.

The construction of $B_{m}$ is based on the Fourier-Bargmann transform which is an isometry from $L_{2}(\mathbb{R})$ into $L_{2}\left(\mathbb{R}^{2}\right)$.

To do this, we consider $h \in L_{2}(\mathbb{R})$ as a windowed function and denote $h^{u, v}(x)=$ $e^{i u x} h(x-v)$. Then we can consider the windowed transform (the Fourier-Bargmann transform or the Gabor Fourier transform):

$$
B_{h}: L_{2}(\mathbb{R}, d x) \longrightarrow L_{2}\left(\mathbb{R}^{2}\right), \quad f \longrightarrow B_{h} f(u, v)=\left\langle f, h^{u, v}\right\rangle_{L_{2}(\mathbb{R})}
$$

In the nice Folland's book [13], the Fourier-Bargmann transform is called wave packet transform and is very close to the Bargmann transform and FBI transform; see also [14].

By taking $h(x)=\left(1 / \pi^{4}\right) e^{-x^{2} / 2}$, Daubechies has pointed out that $B_{h}\left(L_{2}(\mathbb{R})\right)$ is isomorphic to the Bargmann space.

In this work, taking $h_{0}(x)=\left(1 / \pi^{4}\right) e^{-x^{2} / 2}$ and the Hermite functions $h_{m}(x)=$ $\left(1 / \sqrt{2^{m} m !}\right)(x-d / d x)^{m} h_{0}(x)$ as windowed functions, we point out that $B_{h_{m}}\left(L_{2}(\mathbb{R})\right)$ (Gabor space with the $m^{\text {th }}$ Hermite window $h_{m}$ ) is isomorphic to a "generalized" Bargmann space $E_{m}$.

We adopt this last construction to give a "generalized" Bargmann transform $B_{m}$ associated to $E_{m}$ and we construct the phase spaces $I_{m}$ of "generalized coherent states" $|z\rangle_{m}$ associated to $E_{m}$.

To do this, let $z=u+i v$ and $m=0,1, \ldots$, we use the following integral transform:

$$
B_{h_{m}}: L_{2}(\mathbb{R}, d x) \longrightarrow L_{2}\left(\mathbb{R}^{2}, d u d v\right), \quad f \longrightarrow B_{h_{m}} f
$$

such that

$$
\begin{gathered}
B_{h_{m}} f: \mathbb{R}^{2} \longrightarrow \mathbb{C} \\
(u, v) \longrightarrow B_{h_{m}} f(u, v)=\left\langle f, h_{m}^{z}\right\rangle_{L_{2}(\mathbb{R})}=\int_{\mathbb{R}} e^{-i u x} h_{m}(x-v) f(x) d x,
\end{gathered}
$$


where

$$
h_{m}^{z}(x)=e^{i u x} h_{m}(x-v)
$$

We try to be self-contained and elementary as far as possible in this paper which is organized as follows: in Section 2, we present a construction of "generalized" Bargmann spaces following the Hermite functions by using the integral transform (I) for all $m$, and we define the "generalized" Bargmann transform associated to $E_{m}$. In Section 3, we construct the "generalized coherent states" $|z\rangle_{m}, m=0,1, \ldots$, associated to "generalized" Bargmann spaces $E_{m}$ and give some properties of them.

\section{Construction of Generalized Bargmann Spaces Following the Hermite Functions}

Let us consider the Hamiltonian oscillator $H_{\mathrm{osc}}=1 / 2\left(P^{2}+Q^{2}\right)$ on $L_{2}(\mathbb{R})$, where $Q, P$ are the usual position and momentum operators $([Q, P]=i I)$. It is well-known that the eigenvalues and the eigenvectors of $H_{\mathrm{osc}}$ are given by

$$
H_{\mathrm{osc}} h_{m}=\left(m+\frac{1}{2}\right) h_{m}
$$

where $h_{m}$ are the following Hermite functions:

$$
h_{m}(x)=\frac{1}{\sqrt{2^{m} m !}}\left(x-\frac{d}{d x}\right)^{m} \frac{1}{\pi^{4}} \frac{1}{\sqrt{2^{m} m !}} e^{-x^{2} / 2}=\frac{1}{\pi^{4}} \frac{1}{\sqrt{2^{m} m !}} e^{-x^{2} / 2} H_{m}(x)
$$

and $H_{m}(x)=\sum_{j=0}^{[m / 2]}(-1)^{j}(m ! / j !(m-2 j) !)(2 x)^{m-2 j}$ are the Hermite polynomials satisfying the recursion relations $x H_{m}(x)=m H_{m-1}(x)+(1 / 2) H_{m+1}(x)$.

Remark 2.1. (i) It is well-known that $\lambda_{m}=m+1 / 2$ may also be seen as the eigenvectors of the usual Schrödinger operator $H_{S}$ on $\mathbb{R}^{2}$ in presence of a constant magnetic field. Namely $H_{S}$ acting in $L_{2}\left(\mathbb{R}^{2}\right)$ is given by

$$
H_{S}=-\frac{1}{4}\left[\left(\frac{\partial}{\partial x}+i y\right)^{2}+\left(\frac{\partial}{\partial y}-i x\right)^{2}\right] .
$$

(ii) $H_{S}$ has an infinite degeneracy.

(iii) It is well-known that the complex Hermite polynomials

$$
H_{n, k}(z, \bar{z})=(-1)^{m+k} e^{|z|^{2}} \frac{\partial^{m+k}}{\partial z^{m} \partial \bar{z}^{k}} e^{-|z|^{2}}
$$

are an orthogonal basis of the Hilbert space $L_{2}(\mathbb{C}, d \mu(z))$ and we can see in [15] how it was used to give some spectral properties of Cauchy transform on $L_{2}(\mathbb{C}, d \mu(z))$. 
If we formally take $z$ to be real in (2.4), the complex Hermite polynomials $H_{n, k}(z, \bar{z})$ become the well-known real Hermite polynomials $H_{n+k}(x)$.

Now, let $z=u+i v$ and $m=0,1, \ldots$, we define the following integral transforms:

$$
B_{h_{m}}: L_{2}(\mathbb{R}, d x) \longrightarrow L_{2}\left(\mathbb{R}^{2}, d u d v\right), \quad f \longrightarrow B_{h_{m}} f
$$

by

$$
B_{h_{m}} f(u, v)=\left\langle f, h_{m}^{z}\right\rangle_{L_{2}(\mathbb{R})}=\int_{\mathbb{R}} e^{-i u x} h_{m}(x-v) f(x) d x,
$$

where

$$
h_{m}^{z}(x)=e^{i u x} h_{m}(x-v)
$$

(1) If we take in (2.6) $f(x)=h m+k(x)$ for $k=0,1, \ldots$, then we can use the formulas on the Hermite functions given in $[16,17]$, in particular the famous formula:

$$
\int_{\mathbb{R}} h_{m+k}\left(x-\frac{v}{2}\right) e^{-i u x} h_{m}\left(x+\frac{v}{2}\right) d x=\left(\frac{-i}{\sqrt{2}}\right)^{k} \sqrt{\frac{m !}{(m+k) !}} e^{-1 / 4\left[u^{2}+v^{2}\right]}(u-i v)^{k} L_{m}^{k}\left(\frac{|z|^{2}}{2}\right),
$$

where $L_{m}^{k}(t)$ is the Laguerre polynomial defined by the Rodriguez formula as

$$
L_{m}^{k}(t)=\frac{1}{m !} t^{-k} e^{t}\left(\frac{d}{d t}\right)^{m}\left(t^{k+m} e^{-t}\right), \quad t \in \mathbb{R}
$$

The same formula appeared in [18, Chapter 23].

After a simple variable change, the formula (2.8) can be also expressed as

$$
\int_{\mathbb{R}} h_{m+k}(x) e^{-i u x} h_{m}(x+v) d x=\left(\frac{-i}{\sqrt{2}}\right)^{k} \sqrt{\frac{m !}{(m+k) !}} e^{-1 / 4\left[u^{2}+v^{2}-2 i u v\right]}(u-i v)^{k} L_{m}^{k}\left(\frac{|z|^{2}}{2}\right) .
$$

From (2.10), we deduce for $k=0,1,2, \ldots$ that

$$
B_{h_{m}} h_{m+k}(u, v)=\left(\frac{-i}{\sqrt{2}}\right)^{k} \sqrt{\frac{m !}{(m+k) !}} e^{-1 / 4\left[u^{2}+v^{2}+2 i u v\right]}(u+i v)^{k} L_{m}^{k}\left(\frac{|z|^{2}}{2}\right)
$$


or

$$
B_{h_{m}} h_{m+k}(u, v)=\omega(u, v)\left(\frac{-i}{\sqrt{2}}\right)^{k} \sqrt{\frac{m !}{(m+k) !}}(u+i v)^{k} L_{m}^{k}\left(\frac{|z|^{2}}{2}\right)
$$

where

$$
\omega(u, v)=e^{-1 / 4\left[u^{2}+v^{2}+2 i u v\right]}=\omega(z) .
$$

(2) If $0 \leq \mathrm{k} \leq \mathrm{m}$, we have the following formula:

$$
\int_{\mathbb{R}} h_{m-k}(x) e^{-i u x} h_{m}(x-v) d x=\left(\frac{-i}{\sqrt{2}}\right)^{k} \sqrt{\frac{(m-k) !}{m !}} e^{-1 / 4\left[u^{2}+v^{2}+2 i u v\right]}(u-i v)^{k} L_{m-k}^{k}\left(\frac{|z|^{2}}{2}\right) .
$$

That permits us to write for $0 \leq k \leq m$

$$
B_{h_{m}} h_{m-k}(u, v)=\omega(u, v)\left(\frac{-i}{\sqrt{2}}\right)^{k} \sqrt{\frac{(m-k) !}{m !}} e^{-1 / 4\left[u^{2}+v^{2}+2 i u v\right]}(u-i v)^{k} L_{m-k}^{k}\left(\frac{|z|^{2}}{2}\right) .
$$

Denoting $z^{\prime}=u^{\prime}+i v^{\prime}$, then the reproducing kernel $N_{h_{m}}$ of $B_{h_{m}}\left(L_{2}(\mathbb{R})\right)$ can be computed as

$$
N_{h_{m}}\left(u, v, u^{\prime}, v^{\prime}\right)=\left\langle h_{m}^{z}, h_{m}^{z^{\prime}}\right\rangle_{L_{2}\left(\mathbb{R}^{2}\right)} \cdot
$$

Hence we have

$$
N_{h_{m}}\left(u, v, u^{\prime}, v^{\prime}\right)=\int_{\mathbb{R}} e^{-i u x} h_{m}(x-v) e^{i u^{\prime} x} h_{m}\left(x-v^{\prime}\right) d x
$$

After a simple variable change, for example $x=y+v^{\prime}$, then $N_{h_{m}}\left(u, v, u^{\prime}, v^{\prime}\right)$ can be also expressed as

$$
N_{h_{m}}\left(u, v, u^{\prime}, v^{\prime}\right)=\int_{\mathbb{R}} h_{m}(y) h_{m}\left(y+v^{\prime}-v\right) e^{-i\left(u-u^{\prime}\right)\left(y+v^{\prime}\right)} d y .
$$

Hence

$$
N_{h_{m}}\left(u, v, u^{\prime}, v^{\prime}\right)=e^{-1 / 4\left[u^{2}+v^{2}+2 i u v\right]} e^{-1 / 4\left[u^{\prime 2}+v^{\prime 2}-2 i u^{\prime} v^{\prime}\right]} e^{(u+i v)\left(u^{\prime}-i v^{\prime}\right) / 2} L_{m}\left(\frac{\left(u-u^{\prime}\right)^{2}+\left(v-v^{\prime}\right)^{2}}{2}\right)
$$


or

$$
N_{h_{m}}\left(u, v, u^{\prime}, v^{\prime}\right)=\omega(u, v) \overline{\omega(u, v)} e^{(u+i v)\left(u^{\prime}-i v^{\prime}\right) / 2} L_{m}\left(\frac{\left(u-u^{\prime}\right)^{2}+\left(v-v^{\prime}\right)^{2}}{2}\right) .
$$

The reproducing relation in $B_{h_{m}}\left(L_{2}(\mathbb{R})\right)$ can be written as

$$
B_{h_{m}} f(u, v)=\int_{\mathbb{R}^{2}} N_{h_{m}}\left(u, v, u^{\prime}, v^{\prime}\right) B_{h_{m}} f\left(u^{\prime}, v^{\prime}\right) d u^{\prime} d v^{\prime},
$$

then $B_{h_{m}}\left(L_{2}(\mathbb{R})\right)$ is a reproducing space with the reproducing kernel $N_{h_{m}}$.

Introducing the complex structures $z$ and $z^{\prime}$, the above formulas can be rewriten as

$$
\begin{gathered}
B_{h_{m}} h_{m+k}(z)=\omega(z)\left(\frac{-i z}{\sqrt{2}}\right)^{k} \sqrt{\frac{m !}{(m+k) !}} L_{m}^{k}\left(\frac{|z|^{2}}{2}\right), \\
B_{h_{m}} h_{m-k}(z)=\omega(z)\left(\frac{-i \bar{z}}{\sqrt{2}}\right)^{k} \sqrt{\frac{(m-k) !}{m !}} L_{m-k}^{k}\left(\frac{|z|^{2}}{2}\right), \\
N_{h_{m}}\left(z, z^{\prime}\right)=\omega(z) \overline{\omega(z)} N_{m}\left(z, z^{\prime}\right),
\end{gathered}
$$

with

$$
N_{m}\left(z, z^{\prime}\right)=e^{z \overline{z^{\prime}} / 2} L_{m}\left(\frac{\left|z-z^{\prime}\right|^{2}}{2}\right)
$$

We also can see that by changing the variable $z$ into $\sqrt{2} \xi$ and $z^{\prime}$ into $\sqrt{2} \xi^{\prime}$ that $N_{m}\left(z, z^{\prime}\right)$ can be transformed to kernels given in [9, Theorem 2]

$$
K_{m}\left(\xi, \xi^{\prime}\right)=e^{\xi \bar{\xi} \bar{\xi}} L_{m}\left(\left|\xi-\xi^{\prime}\right|^{2}\right)
$$

Now, we can define the "generalized" Bargmann transforms by

$$
B_{m} f(z)=\frac{1}{\omega(z)} B_{h_{m}} f(z)
$$

and the "generalized" Bargmann spaces by

$$
E_{m}=B_{m}\left[L_{2}(\mathbb{R})\right]
$$

Now, the consequences of all the above computations are summarized in the following form. 
Theorem 2.2. (i) $B_{m}\left[L_{2}(\mathbb{R})\right]$ is a subspace of $L_{2}\left(\mathbb{C}, e^{-|z|^{2}} d u d v\right)$.

(ii)

$$
B_{m} h_{m+k}(z)=\left(\frac{-i z}{\sqrt{2}}\right) \sqrt[k]{\frac{m !}{(m+k) !}} L_{m}^{k}\left(\frac{|z|^{2}}{2}\right), \quad k=0,1, \ldots
$$

(iii) For $m=0,1 / \sqrt{k !}(-i z / \sqrt{2})^{k}$ is a basis of classical Bargmann space $E_{0}$.

(iv)

$$
B_{m} h_{m-k}(z)=\left(\frac{-i z}{\sqrt{2}}\right) \sqrt[k]{\frac{(m-k) !}{m !}} L_{m-k}^{k}\left(\frac{|z|^{2}}{2}\right), \quad k=0, \ldots, m .
$$

(v)

$$
\begin{aligned}
B_{m} f(z) & =\frac{1}{\omega(z)} \int_{\mathbb{R}} e^{i u x} f(x)\left(x-v-\frac{d}{d x}\right)^{m} e^{-(x-v)^{2}} d x \\
& =\frac{\sqrt{2^{m} m !}}{\omega(z)} \int_{\mathbb{R}} e^{-1 / 2\left(x^{2}+v^{2}\right)+i \bar{z} x} H_{m}(x-v) f(x) d x
\end{aligned}
$$

(vi)

$$
B_{m} f(i z)=\sqrt{2^{m} m !} \int_{\mathbb{R}} e^{-(z / 2-x)^{2}} H_{m}\left(x-\frac{z+\bar{z}}{2}\right) f(x) d x
$$

Remark 2.3. (i) The operator $A^{*}=-\partial / \partial z+\bar{z}$ is the adjoint of the operator $A=\partial / \partial \bar{z}$ in $L_{2}\left(\mathbb{C}, e^{-|z|^{2}} d u d v\right)$ and $\Delta=A^{*} A$; see for example [19] or [20].

(ii) In $[9,10]$ the "generalized" Bargmann spaces $E_{m}$ are constructed as null spaces of the second-order differential operator $\Delta=-\partial^{2} / \partial z \partial \bar{z}+\bar{z}(\partial / \partial \bar{z})-m I$ in $L_{2}\left(\mathbb{C}, e^{-|z|^{2}} d u d v\right)$ but in this section we have given another construction of these spaces which allows us to construct the "generalized" Bargmann transforms (2.25).

\section{Construction of Generalized Coherent States $|z\rangle_{m}$ Associated to Generalized Bargmann Spaces $E_{m}$}

In the separable Hilbert space $H$ spanned by the eigenvectors $|n\rangle n=0,1,2, \ldots$ of the positive semidefinite number operator $N=a^{*} a$, one can define for each complex number $z=x+i y$

$$
|z\rangle_{m}=e^{-1 / 2|z|^{2}} \sum_{n=0}^{\infty} A^{*^{m}} \frac{z^{n}}{\sqrt{n !}}|n\rangle,
$$

where

$$
A^{*}=-\frac{\partial}{\partial z}+\bar{z}
$$


The space $\mathbf{I}_{m}$ spanned by the set $\left\{|z\rangle_{m}\right\}$ is a separable Hilbert space which is in fact isomorphic to $H$. This space is called the "generalized" space of coherent states. It is closely related to the "generalized" Bargmann space $E_{m}$.

For any $\left|\phi_{m}\right\rangle \in I_{m}$, we can define a function by

$$
\phi_{m}(z)=e^{1 / 2|z|^{2}}\left\langle\phi_{m} \mid z\right\rangle_{m}=\sum_{n=0}^{\infty} A^{*^{m}} \frac{z^{n}}{\sqrt{n !}}\left\langle\phi_{m} \mid n\right\rangle .
$$

As $\int_{\mathbb{C}}\left|\left\langle\phi_{m} \mid z\right\rangle_{m}\right|^{2} d x d y<\infty$, then

$$
\int_{\mathbb{C}}\left|\phi_{m}(z)\right|^{2} e^{-|z|^{2}} d x d y<\infty
$$

Theorem 3.1. Let $A=\partial / \partial \bar{z}$ and $A^{*}=-\partial / \partial z+\bar{z}$, then the following statements hold.

(i) $A A^{*^{m}} f(z)=m A^{*^{m-1}} f(z) ; m=1,2, \ldots$ for any holomorphic function $f(z)$.

(ii) Let $\phi_{m}(z)=e^{1 / 2|z|^{2}}\left\langle\phi_{m} \mid z\right\rangle_{m^{\prime}}$ then $\phi_{m}(z)$ is in the "generalized" Bargmann space $E_{m}$; $m=0,1, \ldots$

(iii)

$$
\begin{array}{r}
|z\rangle_{m}=\sum_{j=0}^{m}(-1)^{j} C_{m}^{j} \bar{z}^{m-j} a^{* j}|z\rangle_{0}, \\
\text { where } a^{*}|n\rangle=\sqrt{n+1}|n+1\rangle \text { and } C_{m}^{j}=m ! / j !(m-j) !
\end{array}
$$

Proof. (i) Let $f(z)$ be holomorphic function; then by applying the commutation relation $\left[A, A^{*}\right]=I$ and the Cauchy-Riemann equation, we deduce that (i) is satisfied for $m=1$ and by induction that

$$
A A^{*^{m+1}} f(z)=A^{*} A A^{*^{m}} f(z)+A^{*^{m}} f(z)=(m+1) A^{*^{m}} f(z) .
$$

(ii) For $m=0$, we have $|z\rangle_{0}=e^{-1 / 2|z|^{2}} \sum_{n=0}^{\infty}\left(z^{n} / \sqrt{n !}\right)|n\rangle$, and $\phi_{0}(z)$ is in the classical Bargmann space $E_{0}$.

For $m \neq 0$ and $\phi_{m}(z)=\sum_{n=0}^{\infty} A^{*^{m}}\left(z^{n} / \sqrt{n !}\right)\left\langle\phi_{m} \mid n\right\rangle$ we have

$$
A^{*} A \phi_{m}(z)=\sum_{n=0}^{\infty} A^{*} A A^{*^{m}} \frac{z^{n}}{\sqrt{n !}}\left\langle\phi_{m} \mid n\right\rangle .
$$


International Journal of Mathematics and Mathematical Sciences

As the function $f(z)=z^{n}$ is holomorphic, we can apply property (i). Premultiplying by $A^{*}$ the equation $A A^{*^{m}} f(z)=m A^{*^{m-1}} f(z)$, we obtain

$$
\begin{aligned}
A^{*} A \phi_{m}(z) & =\sum_{n=0}^{\infty} A^{*} A A^{*^{m}} \frac{z^{n}}{\sqrt{n !}}\left\langle\phi_{m} \mid n\right\rangle \\
& =\sum_{n=0}^{\infty} m A^{*^{m}} \frac{z^{n}}{\sqrt{n !}}\left\langle\phi_{m} \mid n\right\rangle=m \phi_{m}(z) .
\end{aligned}
$$

(iii) Let

$$
\begin{gathered}
a^{*^{j}}|z\rangle_{0}=e^{-1 / 2|z|^{2}} \sum_{n=0}^{\infty} \frac{z^{n}}{\sqrt{n !}} a^{*^{j}}|n\rangle=e^{-1 / 2|z|^{2}} \sum_{n=0}^{\infty}\left(\frac{\partial}{\partial z}\right)^{j} \frac{z^{n}}{\sqrt{n !}}|n\rangle, \\
|z\rangle_{m}=e^{-1 / 2|z|^{2}} \sum_{n=0}^{\infty} A^{*^{m}} \frac{z^{n}}{\sqrt{n !}}|n\rangle .
\end{gathered}
$$

As $\partial / \partial z(\bar{z} f(z))=\bar{z}(\partial / \partial z) f(z)$, we have

$$
A^{*^{m}}=\left(\bar{z}-\frac{\partial}{\partial z}\right)^{m}=\sum_{j=0}^{m}(-1)^{j} C_{m}^{j} \bar{z}^{m-j}\left(\frac{\partial}{\partial z}\right)^{j},
$$

where $C_{m}^{j}=m ! / j !(m-j) !$ Then

$$
|z\rangle_{m}=\sum_{j=0}^{m}(-1)^{j} C_{m}^{j} \bar{z}^{m-j} a^{* j}|z\rangle_{0}
$$

The action of the annihilation operator $a$ on $|z\rangle_{m}$ for $m \neq 0$ is presented below, in the form of a theorem.

Theorem 3.2. (i) $a|z\rangle_{m}=z|z\rangle_{m}-m|z\rangle_{m-1}$.

(ii) There exists an operator $T_{m}$ such that $T_{m}|z\rangle_{m}=z|z\rangle_{m}$.

Proof. (i) Let $|z\rangle_{m}=e^{-1 / 2|z|^{2}} \sum_{n=0}^{\infty} A^{*^{m}} z^{n} / \sqrt{n !}|n\rangle$, where $A^{*}=-\partial / \partial z+\bar{z}$.

By applying the operator $a=z / 2-\partial / \partial \bar{z}$ on $|z\rangle_{m}$, we get

$$
\begin{aligned}
a|z\rangle_{m} & =\frac{z}{2}|z\rangle_{m}-\frac{\partial}{\partial \bar{z}}\left(e^{-1 / 2|z|^{2}} \sum_{n=0}^{\infty} A^{*^{m}} \frac{z^{n}}{\sqrt{n !}}|n\rangle\right) \\
& =\frac{z}{2}|z\rangle_{m}+\frac{z}{2}|z\rangle_{m}-e^{-1 / 2|z|^{2}} \frac{\partial}{\partial \bar{z}}\left(\sum_{n=0}^{\infty} A^{*^{m}} \frac{z^{n}}{\sqrt{n !}}|n\rangle\right) \\
& =z|z\rangle_{m}-e^{-1 / 2|z|^{2}} \sum_{n=0}^{\infty} \frac{\partial}{\partial \bar{z}} A^{*^{m}} \frac{z^{n}}{\sqrt{n !}}|n\rangle
\end{aligned}
$$


Now as $f(z)=z^{n}$ is a holomorphic function and $A=\partial / \partial \bar{z}$, we have $\partial / \partial \bar{z} A^{*^{m}} f(z)=$ $m A^{*^{m-1}} f(z)$ and we get

$$
a|z\rangle_{m}=z|z\rangle_{m}-m e^{-1 / 2|z|^{2}} \sum_{n=0}^{\infty} A^{*^{m-1}} \frac{z^{n}}{\sqrt{n !}}|n\rangle
$$

that is,

$$
a|z\rangle_{m}=z|z\rangle_{m}-m|z\rangle_{m-1}
$$

(ii) In the above property (i) of the present theorem, it is shown that the "generalized" coherent states are not eigenstates of the annihilation operator. Nevertheless, we will show that these "generalized" coherent states can be interpreted as nonlinear coherent states, see for example the theory of Photon-added coherent states used in [21] or the approach of nonlinear coherent states given in [22].

To do this, we have to show that $|z\rangle_{m}$ obeys to the equation

$$
T_{m}|z\rangle_{m}=z|z\rangle_{m}
$$

with a suitable choice for the operator $T_{m}$. Let us now construct the explicit form of $T_{m}$.

From (3.11), we have $z\rangle_{m}=\sum_{j=0}^{m}(-1)^{j} C_{m}^{j} \bar{z}^{m-j} a^{* j}|z\rangle_{0}$, then $\left.z\right\rangle_{m}$ is in the space $F_{m}$ spanned by $\left\{\bar{z}^{m-j} a^{* j}|z\rangle_{0}\right\}_{j=0,1, \ldots, m}$.

By definition, the coherent states $|z\rangle_{0}$ satisfy

$$
a|z\rangle_{0}=z|z\rangle_{0}
$$

Premultiplying the both sides of this equation by $a^{* j}$ leads

$$
a^{*^{j}} a|z\rangle_{0}=z a^{*^{j}}|z\rangle_{0}
$$

Using the commutation relation $\left[a, a^{*}\right]=I$, the above equation is written as

$$
a a^{*^{j}}-j a^{*^{j-1}}|z\rangle_{0}=z a^{* j}|z\rangle_{0} .
$$

which, making use of the identity $\left(a^{*} a+I\right)^{-1} a a^{*}=I$, leads to

$$
\left[a-j\left(a^{*} a+I\right)^{-1} a\right] a^{* j}|z\rangle_{0}=z a^{* j}|z\rangle_{0}
$$

In the following, we introduce

$$
R_{j}=\left(I-j\left(a^{*} a+I\right)^{-1}\right) a
$$


and $P_{j}$ the projection operator on the space spanned by the system $\left\{\bar{z}^{m-j} a^{* j}|z\rangle_{0}\right\}, j=$ $0,1, \ldots, m$

$$
P_{k} \bar{z}^{m-j} a^{* j}|z\rangle_{0}=\delta_{j k} \bar{z}^{m-j} a^{* j}|z\rangle_{0}
$$

where $\delta_{j k}=1$ if $k=j$ and $\delta_{j k}=0$ if $k \neq j$.

Let

$$
T_{m}=\sum_{j=0}^{m} R_{j} P_{j}
$$

Acting $T_{m}$ on $|z\rangle_{m}$ leads to

$$
\begin{aligned}
T_{m}|z\rangle_{m} & =\sum_{j=0}^{m} R_{j} P_{j} \sum_{j=0}^{m}(-1)^{j} C_{m}^{j} \bar{z}^{m-j} a^{*^{j}}|z\rangle_{0} \\
& =\sum_{j=0}^{m}(-1)^{j} C_{m}^{j} R_{j} \bar{z}^{m-j} a^{*^{j}}|z\rangle_{0} \\
& =\sum_{j=0}^{m}(-1)^{j} C_{m}^{j} \bar{z}^{m-j} R_{j} a^{*^{j}}|z\rangle_{0} \\
& =\sum_{j=0}^{m}(-1)^{j} C_{m}^{j} \bar{z}^{m-j} z a^{*^{j}}|z\rangle_{0} \\
& =z \sum_{j=0}^{m}(-1)^{j} C_{m}^{j} \bar{z}^{m-j} a^{* j}|z\rangle_{0}
\end{aligned}
$$

that is,

$$
T_{m}|z\rangle_{m}=z|z\rangle_{m}
$$

To sum up, we have used the Gabor spaces $B_{h_{m}}\left(L_{2}(\mathbb{R})\right)$ with the $m^{\text {th }}$ Hermite window $h_{m}$ to give another construction of generalized Bargmann spaces $E_{m}$ which is different from that given in $[9,10]$ or $[11]$ and have constructed a family of "generalized" Bargmann transforms attached to these spaces. The states $|z\rangle_{m}$ constructed in this work with $m \neq 0$ are nonclassical and can be interpreted as nonlinear coherent states in nonanalytic representation. The spaces $\bigwedge_{m}:=E_{m-1}, m=1,2, \ldots$, are constituted by the polyanalytic functions $\phi(z)$ of order $m$, that is,

$$
\left(\frac{\partial}{\partial \bar{z}}\right)^{m} \phi(z)=0, \quad m=1,2, \ldots
$$


which are not polyanalytic of any lower order and satisfy

$$
\int_{\mathbb{C}}|\phi(z)|^{2} e^{-|z|^{2}} d x d y<\infty
$$

The functions $\phi(z)$ in $\bigwedge_{m}, m=1,2, \ldots$, can be uniquely expressed in the form

$$
\phi(z)=\sum_{0 \leq k \leq m-1} \bar{z}^{k} \sum_{j=0}^{\infty} a_{j, k} z^{j}
$$

Finally, the spaces $E_{m}$ defined in this work are the "true polyanalytic Fock spaces" of [11] or the "generalized Bargmann spaces" of $[9,10]$.

When $m$ is not zero, we give (in a further work) a complete spectral analysis of the nonselfadjoint operator $H_{I}=B^{*}\left(B+B^{*}\right) B$ on $\bigwedge_{m}$ or on $\oplus_{j=1}^{m} \bigwedge_{m}$ (the natural generalization of the Bargmann space, since (3.25) generalizes the Cauchy-Riemann equation $(\partial / \partial \bar{z}) \phi(z)=0)$, where $B=\partial / \partial z$ and $B^{*}=-\partial / \partial \bar{z}+z$.

The restriction of $H_{I}$ on the Bargmann space $E_{0}$ is the Heun's operator and it has been studied in detail in [23].

\section{Acknowledgment}

I congratulate my son Jean-Karim for his dazzling academic performance in college and for those that get in football.

\section{References}

[1] J. R. Klauder and B. S. Skagerstam, Eds., Coherent States-Applications in Physics and Mathematical Physics, World Scientific, Singapore, 1985.

[2] A. Perelomov, Generalized Coherent States and Their Applications, Texts and Monographs in Physics, Springer, Berlin, Germany, 1986.

[3] E. B. Davies, Heat Kernels and Spectral Theory, vol. 92 of Cambridge Tracts in Mathematics, Cambridge University Press, Cambridge, UK, 1989.

[4] E. Nelson, "A quartic interaction in two dimensions," in Mathematical Theory of Elementary Particles (Proc. Conf., Dedham, Mass., 1965), pp. 69-73, MIT Press, Cambridge, Mass, USA, 1966.

[5] B. Simon, The $P(\phi)_{2}$ Euclidean (Quantum) Field Theory, Princeton Series in Physic, Princeton University Press, Princeton, NJ, USA, 1974.

[6] V. Bargmann, "On a Hilbert space of analytic functions and an associated integral transform," Communications on Pure and Applied Mathematics, vol. 14, pp. 187-214, 1961.

[7] A. Intissar, "Étude spectrale d'une famille d'opérateurs non-symétriques intervenant dans la théorie des champs de reggeons," Communications in Mathematical Physics, vol. 113, no. 2, pp. 263-297, 1987.

[8] A. Intissar, "Approximation of the semigroup generated by the Hamiltonian of Reggeon field theory in Bargmann space," Journal of Mathematical Analysis and Applications, vol. 305, no. 2, pp. 669-689, 2005.

[9] N. Askour, A. Intissar, and Z. Mouayn, "Espaces de Bargmann généralisés et formules explicites pour leurs noyaux reproduisants," Comptes Rendus de l'Académie des Sciences. Série I. Mathématique, vol. 325, no. 7, pp. 707-712, 1997.

[10] N. Askour, A. Intissar, and Z. Mouayn, "Explicit formulas for reproducing kernels of generalized Bargmann spaces of $\mathbb{C}^{n}$," Journal of Mathematical Physics, vol. 41, no. 5, pp. 3057-3067, 2000.

[11] N. L. Vasilevski, "Poly-Fock spaces," in Differential Operators and Related Topics, Vol. I (Odessa, 1997), vol. 117 of Oper. Theory Adv. Appl., pp. 371-386, Birkhäuser, Basel, Switzerland, 2000. 
[12] I. Daubechies, Ten Lectures on Wavelets, vol. 61 of CBMS-NSF Regional Conference Series in Applied Mathematics, SIAM, Philadelphia, Pa, USA, 1992.

[13] G. B. Folland, Harmonic Analysis in Phase Space, vol. 122 of Annals of Mathematics Studies, Princeton University Press, Princeton, NJ, USA, 1989.

[14] A. Martinez, An Introduction to Semiclassical and Microlocal Analysis, Universitext, Springer, New York, NY, USA, 2002.

[15] A. Intissar and A. Intissar, "Spectral properties of the Cauchy transform on $L_{2}\left(\mathbb{C}, e^{-|z|^{2}} \lambda(z)\right)$," Journal of Mathematical Analysis and Applications, vol. 313, no. 2, pp. 400-418, 2006.

[16] E. Feldheim, “Développements en série de polynômes d'Hermite et de Laguerre à l'aide des transformations de Gauss et de Hankel," Proceedings of the Koninklijke Nederlandse Akademie van Wetenschappen, vol. 435, 1940.

[17] E. Feldheim, "Relations entre les polynômes de Jacobi, Laguerre et Hermite," Acta Mathematica, vol. 75, no. 1, pp. 117-138, 1942.

[18] M. W. Wong, Weyl Transforms, Universitext, Springer, New York, NY, USA, 1998.

[19] S. T. Ali, F. Bagarello, and G. Honnouvo, "Modular structures on Trace Class Operators and Applications to Landau Levels ," Journal of Physics A , vol. 43, Article ID 105202, 17 pages, 2010.

[20] I. Shigekawa, "Eigenvalue problems for the Schrödinger operator with the magnetic field on a compact Riemannian manifold," Journal of Functional Analysis, vol. 75, no. 1, pp. 92-127, 1987.

[21] T. M. Duc and J. Noh, "Higher-order properties of photon-added coherent states," Optics Communications, vol. 281, no. 10, pp. 2842-2848, 2008.

[22] M. Bagheri Harouni, R. Roknizadeh, and M. H. Naderi, "Spatial confinement effects on quantum field theory using nonlinear coherent states approach," Journal of Physics A, vol. 42, no. 4, Article ID 045403, 12 pages, 2009.

[23] A. Intissar, "Analyse de scattering d'un opérateur cubique de Heun dans l'espace de Bargmann," Communications in Mathematical Physics, vol. 199, no. 2, pp. 243-256, 1998. 


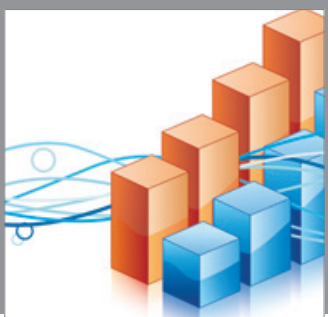

Advances in

Operations Research

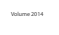

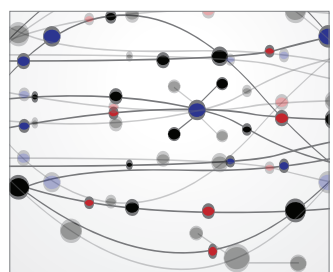

\section{The Scientific} World Journal
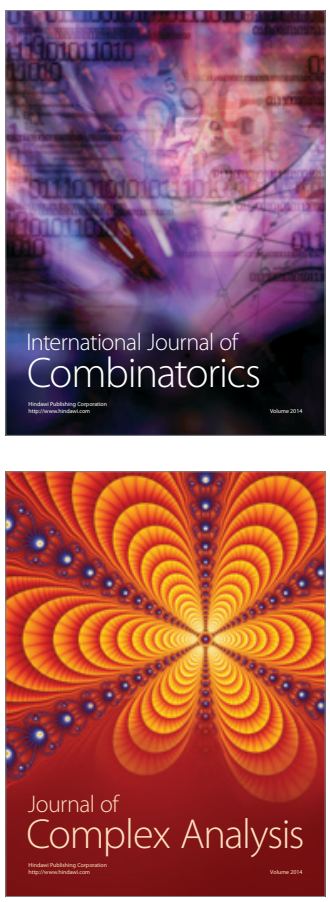

International Journal of

Mathematics and

Mathematical

Sciences
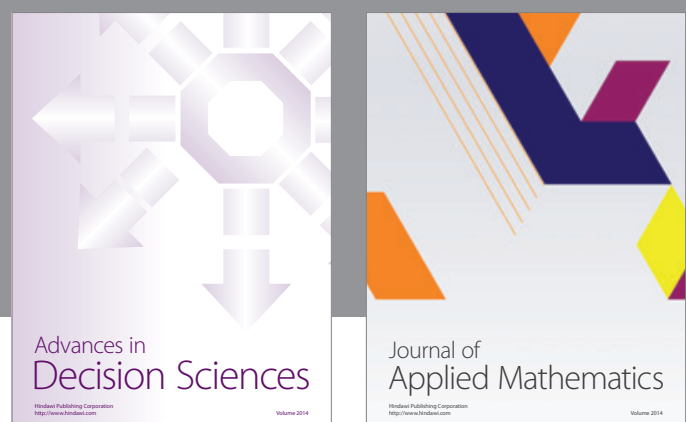

Journal of

Applied Mathematics
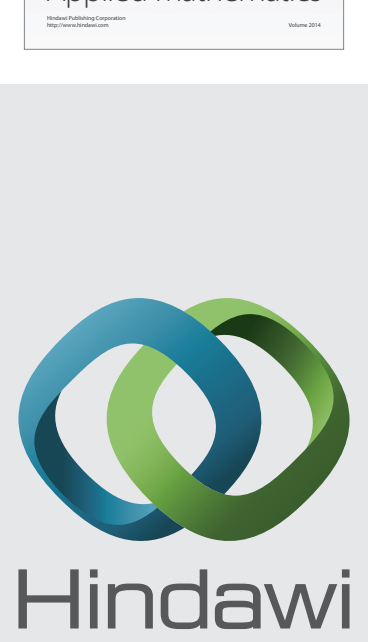

Submit your manuscripts at http://www.hindawi.com
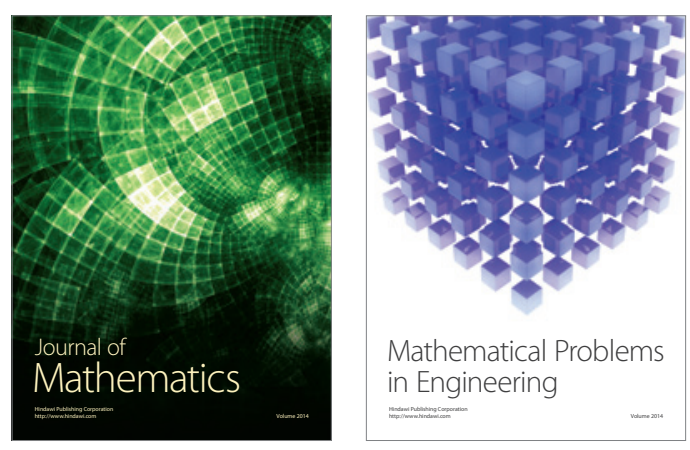

Mathematical Problems in Engineering
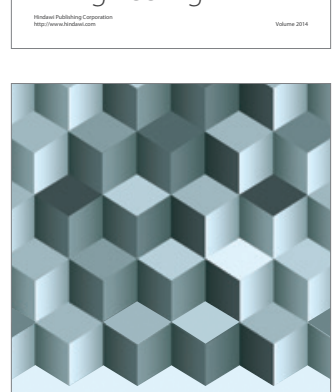

Journal of

Function Spaces
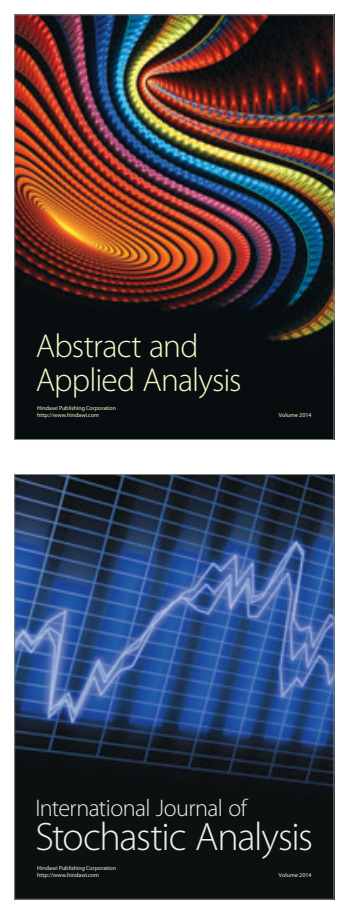

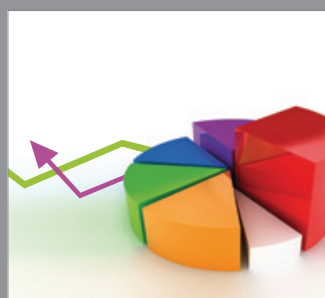

ournal of

Probability and Statistics

Promensencen
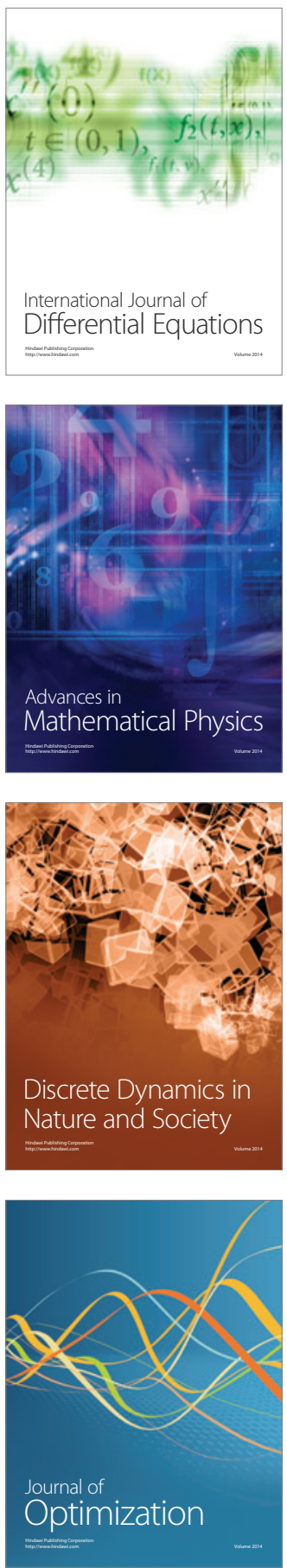